\title{
Perowskit-Solarmodule: Hohe Effizienz auf großer Fläche
}

\author{
Von der Zelle zum Modul ohne Wirkungsgradeinbußen: Das ist eine der wesentlichen \\ Herausforderungen der Perowskit-Photovoltaik. Forschende am Karlsruher Institut für \\ Technologie haben Perowskit-Solarmodule fast ohne Skalierungsverluste hergestellt. Sie \\ erreichten einen Wirkungsgrad von $18 \%$ auf einer Fläche von vier Quadratzentimetern - ein \\ Weltrekord für vakuumprozessierte Perowskit-Solarmodule.
}

In der Photovoltaik gelten Perowskit-Halbleiter dank ihrer günstigen Verfügbarkeit, ihrer einfachen Herstellbarkeit sowie ihres enormen Wirkungsgradpotenzials als besonders zukunftsträchtige Materialien. Perowskit-Solarzellen haben im vergangenen Jahrzehnt eine einzigartige Entwicklung durchlaufen. Im Labor hergestellt erzielen sie inzwischen Wirkungsgrade von über $25 \%$. Der Wirkungsgrad gibt an, wie viel der eingestrahlten Lichtenergie in elektrische Energie umgewandelt wird. Nun gilt es, die Perowskit-Photovoltaik vom Labor in die Industrie zu bringen.

„Eine zentrale Herausforderung besteht darin, die auf Flächen von wenigen Quadratmillimetern erzielten Wirkungsgrade auf typische Solarmodulflächen von einigen Hundert Quadratzentimetern zu übertragen“, sagt Dr. Tobias Abzieher, der am Lichttechnischen Institut (LTI) des KIT die Entwicklung aus dem Vakuum abgeschiedener Perowskit-Solarzellen leitet. Bei PerowskitSolarzellen handelt es sich um Dünnschicht-

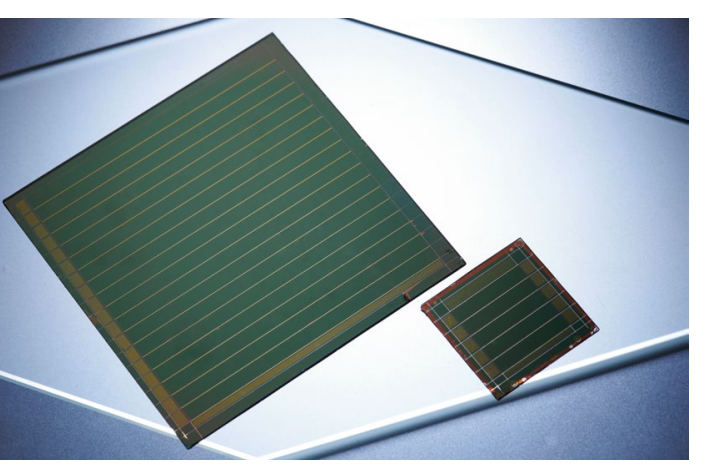

Zellen fast ohne Verluste zu Modulen zu verschalten, gelingt mit einer neuen Kombination von Verfahren. (C Amadeus Bramsiepe, KIT) solarzellen. Diese werden über die sogenannte monolithische Serienverschaltung zu großflächigen Solarmodulen zusammengefügt. Dazu werden während der Abscheidung der einzelnen Schichten der Solarzelle Strukturierungslinien eingebracht, die eine Serienverschaltung der so entstehenden Solarzellenstreifen bewirken.

\section{Schichten der Solarzelle im Vakuum aufgedampft}

Bei Perowskit-Solarmodulen kam es durch die Aufskalierung bisher zu deutlichen Wirkungsgradeinbußen. Erstens ist die Abscheidung der einzelnen Solarzellenschichten umso schwieriger, je größer die Flächen werden, und zweitens entstehen bei der Serienverschaltung Totflächen zwischen den aktiven Solarzellenstreifen. Das sind Flächen, die später nicht zur Stromerzeugung beitragen können, für die Serienverschaltung aber benötigt werden. Dem Karlsruher Team ist es nun gelungen, durch das Aufdampfen aller Schichten der Solarmodule im Vakuum den Einfluss beider Verlustmechanismen zu minimieren. „Große Vorteile der Abscheidung aus dem Vakuum im Hinblick auf die Herstellung effizienter Solarmodule sind die einfache Beherrschbarkeit der Prozesse, die geringe Anzahl an Prozessparametern sowie insbesondere die Unabhängigkeit des Abscheidungsmechanismus von der Beschichtungsfläche", erklärt Abzieher. Dieses Verfahren haben die Forschenden mit der hochpräzisen Strukturierung und Serienverschaltung über das Eingravieren von Linien mittels eines Lasers kombiniert (monolithische Serienverschaltung). Damit haben sie erstmals ein großflächiges Perowskit-Solarmodul fast ohne Skalierungsverluste hergestellt - ein wichtiger Schritt vom Labor in die Industrie.

Mit der Kombination von Vakuumprozessierung und Laserablation erzielten die Forschenden am LTI Wirkungsgrade von bis zu $16,6 \%$ auf einer Bauteilfläche von über $50 \mathrm{~cm}^{2}$ und von sogar $18 \%$ auf einer Fläche von $4 \mathrm{~cm}^{2}$ - ein Weltrekord für vakuumprozessierte Perowskit-Solarmodule. Ihre Ergebnisse präsentierten die Wissenschaftler auf der diesjährigen Frühjahrstagung der Materials Research Society (MRS). „Trotz der Vergrößerung der Bauteilfläche um einen Faktor von über 500 sind fast keine Wirkungsgradeinbußen zu beobachten“, berichtet David Ritzer vom LTI, der die hochpräzise Laserverschaltung entwickelt hat. Mit seinem Ansatz gelang es dem Karlsruher Team, die Skalierungsverluste bei Perowskit-Solarmodulen auf Werte zu senken, wie sie für in der Industrie bereits etablierte Photovoltaiktechnologien wie Cadmiumtellurid (CdTe) oder Kupfer-Indium-Gallium-Diselenid (CIGS) erzielt werden.

Künftig werden die Forschenden an der Optimierung des reinen Solarzellenschichtstapels sowie einer weiteren Reduzierung der Totflächen arbeiten. „Schöpfen wir das Potenzial der Technologie voll aus, ist die Herstellung von Perowskit-Solarmodulen mit Wirkungsgraden von deutlich über $20 \%$ auch auf noch größeren Flächen ein zeitnah realisierbares Ziel“, sagt Professor Ulrich W. Paetzold, Leiter der institutsübergreifenden „Taskforce Perovskite Photovoltaics" am KIT.

Kontakt:

KIT-Zentrum Energie, Karlsruhe, www.energie.kit.edu 